\title{
CONFECÇÃO DE MODELOS ARTIFICIAIS DE BAIXO CUSTO COMO AUXÍLIO APRENDIZAGEM DE ACESSO VASCULAR EM PEQUENOS ANIMAIS
}

\author{
Camila Lopes Ribeiro¹, Caroline Ramos Bittencourt¹, Clara Alita Corona Ponczek ${ }^{1}$ \\ Ivan Roque Barros Filho', Simone Tostes Oliveira ${ }^{1}$
}

\author{
1 UFPR \\ Correspondência: Simone Tostes Oliveira: tostesimone@yahoo.com.br
}

\begin{abstract}
RESUMO: A constante discussão acerca do uso prejudicial dos animais para o ensino está relacionada com a criação e validação de novos métodos ao uso dos animais vivos. O objetivo do trabalho é descrever três modelos artificiais de baixo custo para o treinamento de punção venosa em pequenos animais. Para a construção dos modelos, foi utilizado material de baixo custo e de fácil aquisição, como madeira, tubos de látex, bandagem elástica, esparadrapo e equipos para soro. Os modelos foram criados para representar a realidade em relação ao diâmetro dos vasos, consistência das estruturas e sua proporção em relação ao tamanho antebraço e região cervical. As técnicas de coleta incluíram punção por seringa e agulha ou tubos à vácuo para coleta de sangue, administração de fármacos com seringa e agulha e introdução de cateter para administração de fluido. Os três modelos foram desenvolvidos para contribuir no aprendizado das técnicas de punção venosa e não substituem a técnica em animais vivos, mas auxiliam os profissionais, acadêmicos de medicina veterinária e enfermeiros veterinários antes das primeiras coletas. Este treinamento prévio aumenta a confiança do profissional, além de não haver a possibilidade de contato com material infectante. $O$ modelo, sendo barato, de preparo simples e rápido, e com material de fácil aquisição, pode ser confeccionado por qualquer profissional, servindo como alternativa de aprendizagem para o uso de animais no ensino.
\end{abstract}

Palavras-chave: cães; coleta de sangue; ensino; gatos; métodos alternativos

\section{INEXPENSIVE HOMEMADE MODELS FOR VASCULAR ACCESS TRAINING IN SMALL ANIMAL PRACTICE}

\begin{abstract}
The constant discussion about the harmful use of animals in education is related to the creation and validation of new alternative methods to the use of live animals. The aim of this work is to describe three inexpensive artificial models for training venipuncture in small animals. Cheap and easily acquired material were used in the building of the models, such as wood, latex tubes, elastic bandaging tape, adhesive tape, and intravenous administration set. The models were created to represent reality in terms of vessel diameter, consistency of structures and proportion to the forearm and neck. The collection techniques included venipuncture using syringe for blood collection and administration of drugs; vacuum tubes for blood collection; and catheter insertion for fluid administration. The three models were developed to help students in learning the techniques of venipuncture and are not substitutes for practice in living animals. However, they help professionals, veterinary medicine students and veterinary technicians in their first contact with venipuncture techniques. This previous training increases professional confidence. Moreover, there is no possible contact with infectious material. These cheap, simple and easy to prepare models may be made by any professional, serving as an alternative to the use of animals in education.
\end{abstract}

Key Words: alternative methods; blood collection; cat; dogs; teaching 

vascular em pequenos animais

\section{INTRODUÇÃO}

A coleta de sangue para realização de exames é indispensável na Medicina Veterinária, e a punção correta do vaso sanguíneo é necessária para garantir a qualidade das amostras e minimizar o desconforto do paciente durante a coleta. Entretanto, muitos alunos não recebem treinamento para tal e acabam coletando sangue ou introduzindo um cateter venoso pela primeira vez, em um paciente real. Realizar este tipo de treinamento em cães ou gatos mantidos para experimento seria igualmente imprudente, pois ocasionaria seu sofrimento da mesma forma. Tendo isso em vista, devem-se buscar alternativas ao uso de animais no ensino, utilizando modelos para treinamento do acesso vascular.

A punção venosa em cães ou gatos pode ser realizada nas veias cefálica, jugular, femoral ou safena lateral. Em cães de grande porte, a veia cefálica é a preferida. Nos gatos e nos cães de pequeno porte, é frequentemente indicada a veia jugular (Lopes, 2009). A veia cefálica acompanha a borda cranial do antebraço, onde repousa sobre 0 músculo extensor radial do carpo, e no braço segue entre os músculos peitoral superficial e cleidobraquial do braquiocefálico unindo-se à veia jugular externa na parte inferior do pescoço (Dyce, 2004). A veia cefálica é a escolha mais frequente para canulação venosa, podendo ser palpada quando é levantada pela pressão sobre o cotovelo (Lopes, 2009). A veia jugular externa adota um trajeto pelo pescoço entre os músculos braquiocefálico (parte cleidocervical) e esternocefálico. Os músculos esternocefálico e braquiocefálico são separados caudalmente, o que permite que a veia jugular externa se torne subcutânea (Dyce, 2004).
Ainda que produtos alternativos, incluindo modelos, simuladores, softwares e internet já estejam disponíveis para treinamentos diversos nos cursos de Biologia, Medicina, Enfermagem e Medicina Veterinária (Jukes e Chiuia, 2003), o número de modelos existentes para a Medicina Veterinária ainda é insuficiente (Scalese e Issenberg, 2005). Assim como no exterior, algumas faculdades de Medicina Veterinária no Brasil tem se empenhado no desenvolvimento e uso de recursos alternativos em suas aulas práticas e no uso ético de cadáveres (Dinóla e Roncati, s.d.; Matera, 2008; Tudury e Potier, 2009; Costa Neto e Martins Filho, 2011). Diversos métodos humanitários já foram comparados aos métodos convencionais, sendo em sua maioria equiparáveis em termos de conhecimentos ou habilidades obtidos pelo aluno (Greif, 2003; Naylor et al., 2009). A busca por recursos alternativos ao uso de animais leva à valorização da ética no ambiente educacional, além de preservar a integridade ética, moral, psicológica e social dos alunos (Magalhães e Ortêncio Filho, 2006).

O objetivo do trabalho é apresentar modelos artificiais de baixo custo como auxilio didático às diversas técnicas de punção venosa em pequenos animais, auxiliando profissionais e, principalmente, graduandos, antes de uma primeira coleta, proporcionando que os mesmos conheçam os materiais utilizados e a técnica correta, evitando que os animais sejam manipulados por pessoas inexperientes ou de forma desnecessária.

\section{MATERIAL E MÉTODOS}

Foram confeccionados três modelos artificiais, sendo que um retrata o antebraço do cão e, os outros dois, o pescoço. Os modelos foram elaborados para o treinamento de punção venosa com seringa e agulha para coleta de 
sangue e administração de fármacos; punção com tubo a vácuo para coleta de sangue e colocação de cateter para administração de fluido.

Para o modelo artificial do antebraço foram utilizados madeira, tubo de látex para garrote, bandagem elástica, equipo, frasco de solução fisiológica, corante alimentício e esparadrapo. As etapas da montagem estão ilustradas na figura 1. Primeiramente, foi utilizada a madeira $(4 \times 4 \times 20 \mathrm{~cm})$ com sulco de $0,5 \mathrm{~cm}$, onde foi encaixado o tubo de látex para garrote que simula a veia cefálica $(0,5$ $\mathrm{cm}$ de diâmetro $\times 25 \mathrm{~cm}$ de comprimento). A seguir, foi fixado o tubo de látex no sulco da madeira com esparadrapo, envolvendo a madeira com bandagem elástica de $12 \mathrm{~cm}$ de largura. Foi acoplado ao tubo de látex um equipo seccionado em sua porção final, sendo cada parte deste acoplada em cada lado do tubo de látex, com a extremidade dobrada e fixada com esparadrapo para não haver vazamento do fluido. O equipo foi acoplado a um frasco de solução fisiológica, sendo adicionado corante alimentício de coloração vermelha, para melhor visualização do líquido durante as punções.

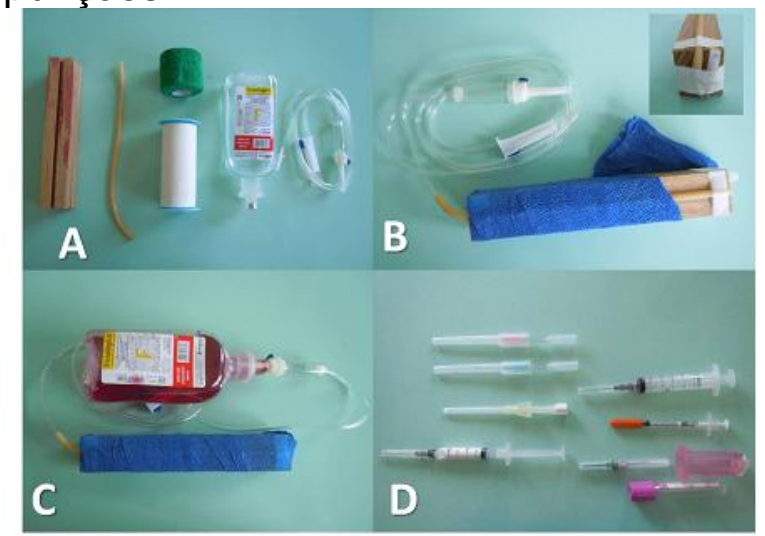

Figu ra 1 - Etapas da confecção de modelo artificial de antebraço para técnica de punção venosa em pequenos animais.

A-material utilizado. B-montagem do modeb, dobrando-se a ponta contralateral do equipo (detalhe). C-modelo pronto; observar que a ponta do equipo foi escondida passando-se bandagem. D-material utilizado nos testes para treino da puncäo: passando-se bandagem. D- material utilizado nos testes para tre ino da punção:
seringas de 5,3 e $1 \mathrm{~mL}$ para coleta de "sangue" e para aplicaçăo de medicamentos, cateter 24,22 e 20, tubo de coleta à vácuo.
Para simular a punção da veia jugular, foram confeccionados dois modelos de pescoço, sendo um modelo para acesso da região cervical com o animal em decúbito esternal ou sentado, e o outro em decúbito lateral. Para a confecção do pescoço em decúbito esternal ou sentado, os materiais utilizados foram isopor, espuma, fio de náilon, agulha, traqueia corrugada, sonda de Foley ou garrote, equipo, bandagem elástica, frasco de soro, corante alimentício, película de raios- $X$ e papel contact (plástico autoadesivo). As etapas da montagem estão descritas na figura 2. Foi utilizado isopor $(6 \times 30 \times 4$ $\mathrm{cm})$, ao redor do qual foi enrolada uma espuma $(32 \times 30 \mathrm{~cm}$ - densidade 20$)$ para simular a musculatura cervical. A espuma foi costurada em sua linha média, configurando um formato cilíndrico. Foi colocada uma traqueia corrugada $(12 \mathrm{~cm})$ na linha de costura para simular a traqueia e, para simular a veia jugular, foi colocada paralelamente à traqueia uma sonda de Foley $14 \mathrm{Fr}$ ou um tubo de látex (garrote). Foi acoplado o equipo na extremidade bifurcada da sonda de Foley com frasco de soro. Posteriormente foram envolvidos todos os materiais na bandagem elástica. $O$ pescoço foi fixado a uma base cônica feita de película para raios- $X$ e recoberta por papel contact.

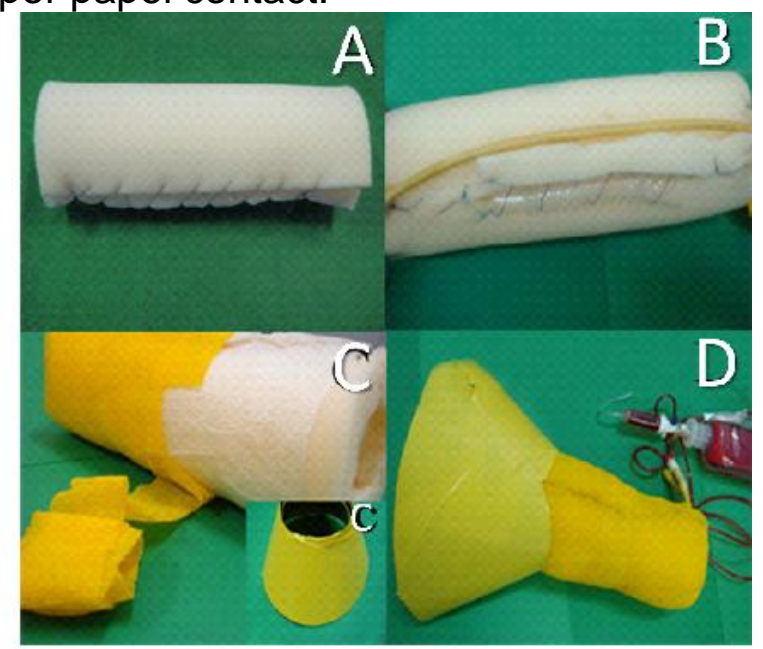

Figura 2 - Etapas da montagem do modelo artificial de pescoço simulando animal em decúbito esternal ou sentado.

A a C-confecção do modelo, notar película de raios-x recoberta por papel contact (c). D-modelo pronto. 

vascular em pequenos animais

Para a confecção do modelo de pescoço em decúbito lateral, foram utilizados: tampa de plástico ou pote raso $(30 \times 16,5 \mathrm{~cm})$, traqueia corrugada, espuma (densidade 20), fio de náilon, agulha, sonda de Foley 14Fr, bandagem elástica, equipo, frasco de soro e corante alimentício. As etapas da montagem estão mostradas na figura 3 .

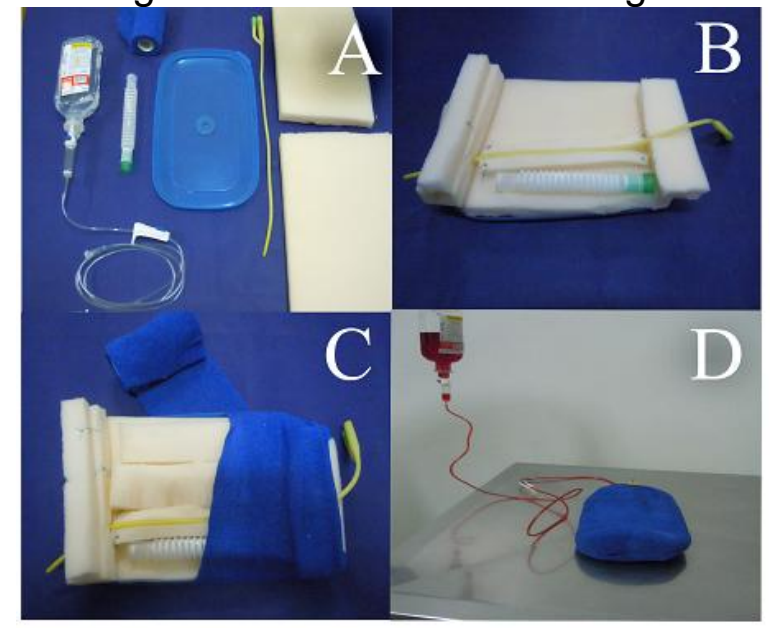

Figura 3 - Etapas da montagem do modelo artificial de pescoço simulando animal em decúbito lateral.

A- Materiais utilizados; B e C- processo de montagem; D- dispositivo pronto.

\section{RESULTADOS E DISCUSSÃO}

Os modelos possibilitaram a punção com seringa e agulha e com tubos a vácuo, assim como a introdução de cateteres e a simulação de administração de fármacos (Figura 4). Tanto a sonda de Foley quanto o tubo de látex para garrote se mostraram eficientes para as técnicas de coleta de sangue, com seringa ou tubos a vácuo, e para introdução de fármacos com seringa. A sonda de Foley não permitiu a introdução de cateter, mostrando resistência à passagem dos cateteres 24 e 22, danificando o teflon. O tubo de látex possibilitou a passagem dos cateteres com resistência semelhante a do vaso, permitindo a fácil introdução de todos os cateteres testados. Uma opção, que serve aos mesmos propósitos ao tubo de látex, seria a utilização de equipo comprado já com a borracha (adaptador para agulha ou cateter). Neste caso, a única limitação seria 0 fato de a punção ter que ser executada exatamente no ponto onde se encontra o segmento de látex.

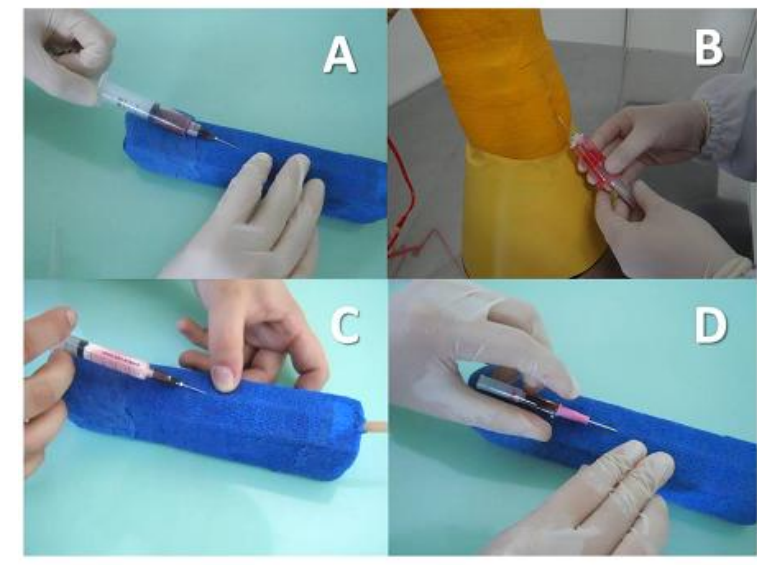

Figura 4 - Técnicas de punção venosa nos modelos artificiais.

A- simulação de coleta de sangue com seringa e agulha na veia cefálica. B-simulação de coleta de sangue com tubo a vácuo na veia jugular. C- introdução de fármaco na veia. DSimulação de introducão de cateter na veia cé́lica.

Para a saída adequada do fluido nos três modelos durante o acesso vascular, bastou manter o frasco de soro elevado, preso a uma haste de soro, e abrir o dispositivo de fechamento do equipo (pinça rolete ou clipe), quando realizada a punção, para que todas as técnicas descritas fossem satisfatoriamente executadas. Da mesma forma, o equipamento não apresentou vazamento e pode ser guardado montado, apenas com 0 cuidado de se fechar o equipo. Quando o conteúdo do frasco termina, basta substituí-lo por outro cheio.

Os modelos foram testados tanto por estudantes do segundo ano do curso de Medicina Veterinária, sem experiência, quanto por alunos do quinto ano, que já apresentavam alguma experiência prévia de coleta de sangue e colocação de cateter em animais. A técnica foi bem aceita por ambos os grupos. Os alunos do segundo ano tiveram um primeiro contato com as técnicas de acesso vascular, e se sentiram confortáveis por treinar nos modelos quantas vezes o desejaram. Disseram se sentir mais seguros e preparados para uma posterior coleta 
em um paciente real. Os estudantes do quinto ano, que já haviam coletado com seringa e agulha em animais antes, relataram que a coleta à vácuo, técnica desconhecida por eles, se tornou mais fácil de ser realizada com treinamento prévio, por possibilitar maior habilidade prática. Também relataram achar válida a utilização dos três modelos, ao invés de um único modelo. Apesar de que, teoricamente, apenas um modelo, como por exemplo, o antebraço artificial, seria suficiente para testar todas as técnicas, os estudantes se sentiram desafiados a treinar o acesso à "jugular", considerada por eles como um vaso de acesso mais difícil do que a veia cefálica. O último autor deste trabalho teve a oportunidade de acompanhar os alunos do quinto ano durante o estágio curricular destes, e acompanhou as várias técnicas de punção venosa, por eles executadas, em pacientes (cães e gatos) da rotina hospitalar. Foi percebido um menor percentual de erros de técnica, assim como melhor localização dos vasos e maior segurança e habilidade dos indivíduos que fizeram o treinamento nos modelos artificiais, em relação a indivíduos no mesmo nível de graduação, que não fizeram o treinamento, ainda que ambos já tivessem experiência de coletas prévias em animais.

Os três modelos foram de fácil e rápida confecção, mimetizando as estruturas anatômicas, podendo ser elaborados por qualquer pessoa, sendo estudantes ou professores. $O$ custo aproximado de cada modelo foi de sete dólares (14 reais). Apesar de que bons modelos artificiais para treinamento vascular em pequenos animais existam, simulando inclusive os dedos e a face do cão, seu custo é elevado, sendo 195,00 a 466,00 dólares o preço de um antebraço (University of California;

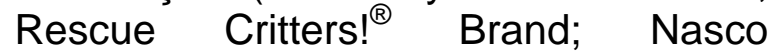
Life/form ${ }^{\circledR}$ ) e 600,00 dólares o de um pescoço de cão (University of
California). Estes custos ainda são limitantes para várias instituições de ensino, incluindo várias universidades públicas no Brasil. Os modelos aqui apresentados tem a vantagem de ser de baixíssimo custo, além de ser de fácil confecção, facilitando desta forma sua fabricação caseira, sem a necessidade de se comprar o modelo pronto. Os modelos atingiram o mesmo objetivo proposto pelos modelos comerciais mais elaborados, que seria o treinamento de acesso vascular para várias técnicas em pequenos animais.

\section{CONCLUSÃO}

Os modelos criados mostraram-se eficazes para o treinamento de punção venosa para coleta de sangue, com seringa e agulha ou tubos de coleta à vácuo, introdução de medicamentos com seringa, assim como para a colocação de cateter intravenoso. Esses dispositivos foram confeccionados artesanalmente a baixo custo, podendo ser usados não apenas no aprimoramento destas técnicas por médicos veterinários, mas também no ensino de técnicas hospitalares e de enfermagem para alunos da graduação.

\section{REFERÊNCIAS}

COSTA NETO, J.M.; MARTINS FILHO, E.F. Uso de animais para o ensino da cirurgia na medicina veterinária. Qual a alternativa? XIX

Seminário Nacional de Ensino da Medicina Veterinária. Seminário. 14 a 16 de setembro de 2011. Brasília (DF). Disponível em:

<http://www.cfmv.gov.br/portal/inscricao_df/mate $\mathrm{rial} /$ dia $>$. Acesso em: 30/12/2012.

DINÓLA, C.; RONCATI, N. A. Utilização de manequins no processo de ensino e aprendizagem da Escola de Medicina Veterinária. Disponível em: $<$ http://portal.anhembi.br/publique/Acontece/Autilizacao-de-manequins-no-processo-deensino-e-aprendizagem-da-Escola-de-MedicinaVeterinaria>. Acesso em: 03/01/2013. 
DYCE, K.; SACK, W.; WENSING, C. Tratado de anatomia veterinária. 3 ed. Rio de Janeiro:

Elsevier: 2004, $813 \mathrm{p}$.

GREIF, S. Alternativas ao uso de animais vivos na educação pela ciência responsável. São Paulo : Instituto Nina Rosa, 2003. 175p.

UNIVERSITY OF CALIFORNIA- UCDavis Veterinary Medicine. Educational Products. Vascular Access Training Models. [s.d] Disponível em:

<http://www.vetmed.ucdavis.edu/products/vatm. cfm> Acesso em 10/12/2012.

JUKES, N.; CHIUIA, M.. From guinea pig to computer mouse: alternative methods for a progressive, humane education. 2nd ed. Leicester: InterNICHE, 2003. 524p.

LOPES, R.D. Manual para coleta de sangue venoso em caninos e felinos. 2009. São Paulo, 71f. Monografia (Especialização em Patologia Clínica Veterinária) -Faculdade de Medicina Veterinária e Zootecnia, Universidade de São Paulo

MAGALHÃES, M; ORTÊNCIO FILHO, $\mathrm{H}$. Alternativas ao uso de animais como recurso didático. Arquivos de Ciências Veterinárias e Zoologia da UNIPAR, v.9, n.2, p.147-154, 2006.

MATERA, J.M. O ensino de cirurgia: da teoria à prática. Ciência Veterinária nos Trópicos, v.11, supl. 1, p.96-101, 2008.

NASCO LIFE/FORM CANINE IV LEG. [s.d] Disponível em:

<http://www.enasco.com/product/LF01016U>. Acesso em: 03/01/2013.

NAYLOR, R.A.; HOLLETT, L.A.; VALENTINE, R.J. et al. Can medical students achieve skills proficiency through simulation training? The American Journal of Surgery, v.198, n.2, p.277-282, 2009.

RESCUE CRITTERS BRAND. Animal Training manikins. [s.d] Disponével em:

<http://www.rescuecritters.com/products/advanc ed-veterinary-training/1-advanced-veterinarytraining/15-k-9-iv-trainer>. Acesso em: 03/01/2013.

SCALESE, R.J.; ISSENBERG, S.B. Effective use of simulations for the teaching and acquisition of veterinary professional and clinical skills. Journal of Veterinary Medical

Education, v.32, n4, p.461-467, 2005.

TUDURY, E. A.; POTIER, G. M. Métodos substitutivos ao uso de animais vivos no ensino: métodos alternativos para aprendizado prático da disciplina técnica cirúrgica veterinária.
Ciência Veterinária nos Trópicos, v.11, supl.1, p.92-95, 2008. 